\title{
Improving nursing methods by using thermal imaging: observations by CAT S60 mobile phone
}

\author{
Jarmo Alametsä ${ }^{1}$, Markku Oikarainen ${ }^{1}$, Jari Viik ${ }^{2}$, Jarmo Perttunen ${ }^{1}$
}

${ }^{1}$ Tampere University of Applied Sciences, Tampere, Finland; ${ }^{2}$ BioMediTech Institute and Faculty of Biomedical Science and Engineering, Tampere University of Technology, Tampere, Finland

Jarmo Alametsä, Tampere University of Applied Sciences, Kuntokatu 3, FI-33520 Tampere, FINLAND. Email: jarmo.alametsa@health.tamk.fi

\begin{abstract}
The purpose of this preliminary study was to examine, how thermal imaging could help in advancing nursing methods and offer some new usage targets of thermal imaging in promoting the wellness of a patient. By using CAT S60 cellular phone with an inbuilt Flir thermal camera, thermal pictures were taken from four voluntary subjects in order to find out if thermal imaging with CAT S60 phone could help the treatment of a patient. Thermal camera images were taken in order to find out temperature changes from the whole body, limbs and extremities. By using thermal imaging the beginning of treatment could be hastened and the monitoring of the state of a patient would be more efficient thus improving the prognosis of a patient. The benefit of the method is, that it is non-invasive, cheap and easy to use (inside a cellular phone) thus being a clear advantage in health care operations.

The results of different usage methods seen in thermal images suggest that thermal imaging with CAT S60 phone could be used to improve nursing methods and may also for its part to help in diagnosis. The present preliminary observations via thermal images showed, that the resolution of CAT S60 phone was sufficient to detect changes in human body temperature in home life. This may suggest the usage of the CAT S60 phone in home care services.
\end{abstract}

Keywords: thermal imaging 


\section{Introduction}

There is a growing need to find a non-invasive, cheap, portable and easy method for accurate skin, limb and body temperature measurement. Thermal imaging $[1,2]$ as a non-invasive method offers easy and quick method to examine cardiovascular system. Thermal imaging reveals the body surface temperature by measuring the emitted infrared thermal radiation forming a digital image of the measured temperatures [1]. Medical doctors have used infrared technology over 100 years time started from the discovery of thermometer in 1871 which lead to the modern infrared thermal imaging methods [1]. Infrared imaging was used in the first time in 1959 to diagnose arthritis revealing unusually high temperatures in patients having joint inflammation [1]. The purpose of the blood circulation along with the transportation of oxygen, waste products and nutrients is to convey heat thus acting as a part of thermoregulation.

The metabolic syndrome (MS) includes as a cluster of risk factors including obesity, hypertension, impaired glucose tolerance and dyslipidemia. The MS is also associated with the development of diabetes and cardiovascular diseases [3]. Thus the condition of the cardiovascular system (along with the aging) contributes to its effectiveness in thermoregulation. The elevated amount of fat in the body is a risk factor for thrombosis $[4,5]$. Thermal imaging has been used to detect breast cancer $[6,7]$, it has been used in intra-operative imaging [8], in ocular imaging [9] and in the screening of fever [7]. It has been used to detect vascular disorders from diabetic subjects (varicose veins with probable mild inflammation), in dental diagnosis, in arterial blood pressure monitoring (hypothermia was observed on the hands of the subjects due to increased blood pressure), in the diagnosis of rheumatic diseases, in the diagnosis of dry eye syndrome and ocular dis-eases, changes in heart operation (in detecting earliest signs of atherosclerosis thus acting as an early sign of heart attack) [7]. Thermal imaging can reveal temperature variations as a first symptom before the onset of harmful tissue changes and problems in blood circulation. It has been used in dermatological diseases, with diabetes mellitus patients having foot ulceration and inflammatory state studies [2]. It has been even utilized in gynecology [7]

In temperatures $27-32 \mathrm{C}^{\circ}$ naked, resting human has a thermoneutral zone (comfort zone). In this area the regulation of skin circulation is sufficient to maintain the heat loss stable during the ambient temperature change. The human core temperature stays in $37 \mathrm{C}^{\circ}$. The skin insulation properties get better when the small skin arteries constrict (vasoconstriction) and blood circulation de-creases. During body cooling the motoric nerve fibers in muscles activate causing rhythmic involuntary constriction (myokymia) having a frequency of 10-20 constrictions in second. During muscle constriction muscles constrict to the same time; visible movement is not seen and muscle work is not done, but the whole increase in muscle cell metabolism converts almost completely to the heat. It is so efficient, that the heat production of the body can be multiplied in a few seconds. The body temperature decrease below $25 \mathrm{C}^{\circ}$ may lead to ventricular fibrillation [10]. Myocardial infarction and cardiovascular (CV) related mortality increase in low outdoor temperature [11].

The objective of this study was to explore whether CAT S60 cellular phone would introduce some extra value for example to the home care workers as a tool for recording changing patient thermal values. This would be useful in situations, where the total human body temperature needs to be measured or to look out the temperature of the limbs, for example in situations, where an elderly person is found outdoor. Heat traces can also reveal when the person left the site very easily thus providing vital information for example for the search of disappeared people. Especially here in Finland, where outdoor temperatures may be very low in winter, the exact situation of the limb or body temperature is vital revealing body parts which may get frostbitten. The quick detection of the cooling limb revealing poor blood circulation may accelerate treatment operations and may advance patient prognostication. In medical practice the inspection of a wound is mainly based of the eyesight of the physician. There are no standardized instruments to evaluate the moisture of a wound, the swelling of the surrounding healthy tissue or colour or the amount of the granulation tissue in the basis of 
the wound. The physician's base of reference evolves via hundreds or thousands cases of wounds [12]. The evaluation of the appearance of the wound is the base for wound treatment defining the proper materials for wound care. The main problem is that it is based on visual perception [12] and thus is prone to errors. In wound healing thermal imaging may give systematic extra information about the amount of tissue, the edges of a wound, the level of infection and moisture of the wound. This extra information may accelerate the process of wound evaluation and the beginning of proper healing methods.

\section{Methods and measurements}

CAT S60 cellular phone with an inbuilt Flir thermal camera with MSX technology was used to obtain thermal pictures from four voluntary people on condition revealing the state of local circulation seen as radiated heat from the different part of the body.

Flir thermal pictures were taking in home conditions (during fall 2016); in a private house (figures 1 - 2) and in a residential home (figures 3-5). There were four participants in the study and the age distribution was from 30 to 70 years of age. The age of the person in figures 1-2 was 51 years, in figure 370 years, in figures 4-5 60 years and in figure 6 about 30 years. Figure 6 was taken during a 'Restart a heart' day in a mall, where students were performing a recovery exercise with a doll. The pictures were taken in order to improve nursing methods and to offer suggestions, where thermal imaging may give a hint what is wrong with the patient.

\section{Results}

The obtained thermal images with CAT S60 phone showed, that the resolution of the thermal images is sufficient for the study. In figure 1 the heat dissipation due to sauna bath increased through the whole body. In figure 2 some differences were observed in heat dissipation between left and right side of the back. In figures 3-5 temperature differences were observed especially in the extremities like in toes and fingers with older people having decreased level of circulation in the extremities. 


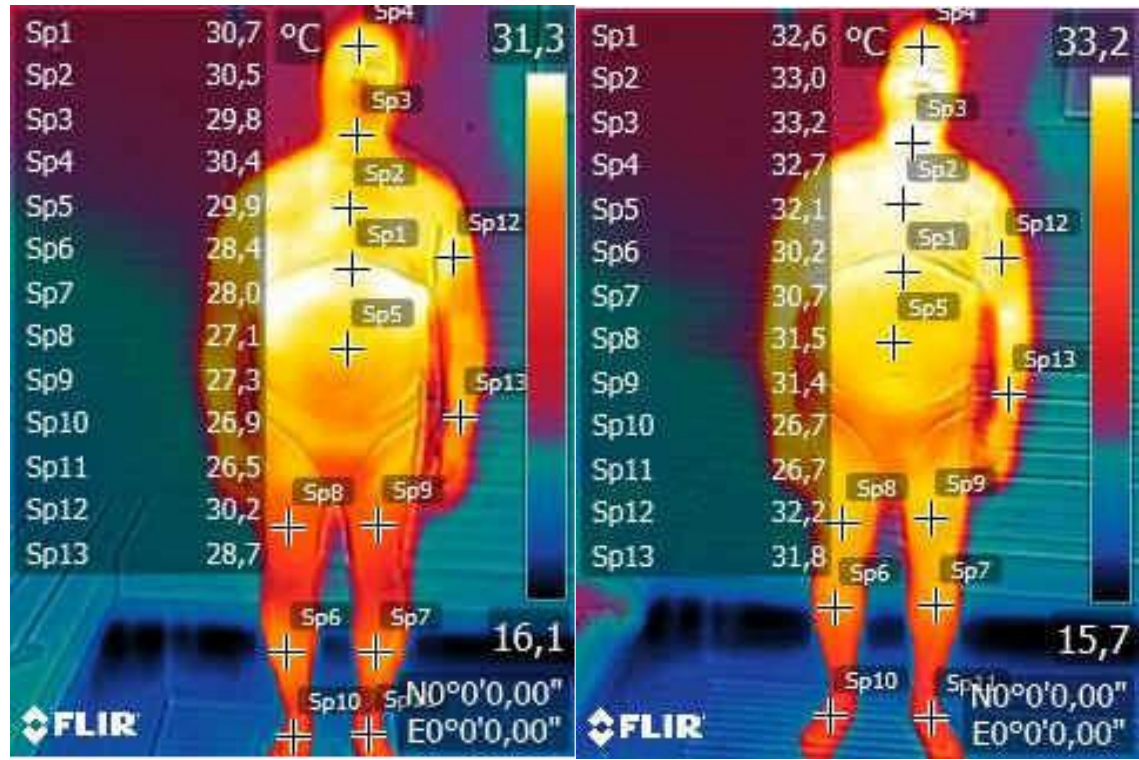

Figure 1. Thermal image from the whole body from a person before the sauna bath on the left taken with CAT S60 phone. The heat dissipation from the limbs seems similar. In the picture on the right there is a situation after sauna bath. Heat dissipation has increased due to sauna bath (difference from $0,2 C^{\circ}$ to $4,4 C^{\circ}$ ). In the location of thigh muscle (Sp8 on the right; difference 4,4 $\mathrm{C}^{\circ}$ and Sp9 on the left; $4,1 \mathrm{C}^{\circ}$ ) was the highest heat difference. The lowest heat dissipation was in the location of dorsalis pedis (Sp10 and Sp11; difference $0,2 \mathrm{C}^{\circ}$ ). In the neck region heat dissipation was larger (Sp3; difference 3,4 $\mathrm{C}^{\circ}$ ) due to vicinity of arteries. In the ankle heat dissipated also (Sp6 on the right; difference $1,8 \mathrm{C}^{\circ}$ and Sp7 on the left; difference $2,7 \mathrm{C}^{\circ}$ ). The measured person had earlier slightly elevated blood pressure (BP) values, which have been treated with sauna bath [13]. Nurses assist patients also in sauna bath.

A

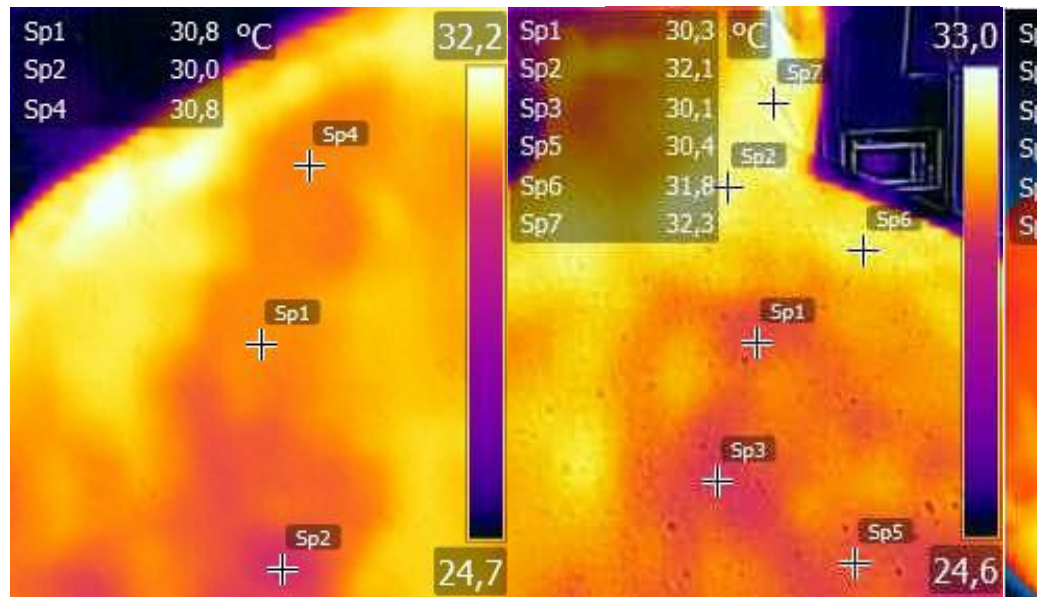

C

Sp1

\section{Sp1}

Sp2

Sp3.

Sp4

Sp5.

Sp6.

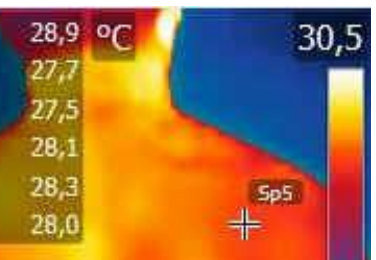

Sp2 $5 p 2$
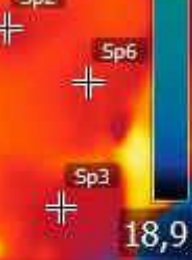

Figure 2. Picture from a person on the left side $(A)$ and on the right side $(B)$ of the back having pain in the right back and the right shoulder. In colder areas the blood circulation ability of the veins might have decreased or disturbed. This may be due to local inflammation. C) Picture from the back after several months from the picture taken (A, B). On the right side of the back seems to have colder spots when compared with the left side of the back. Occasional pain was present in near Sp3 location. Thermal imaging has been used in lower back pain studies, where a body sur-face temperature map may give additional diagnostic knowledge [2]. 

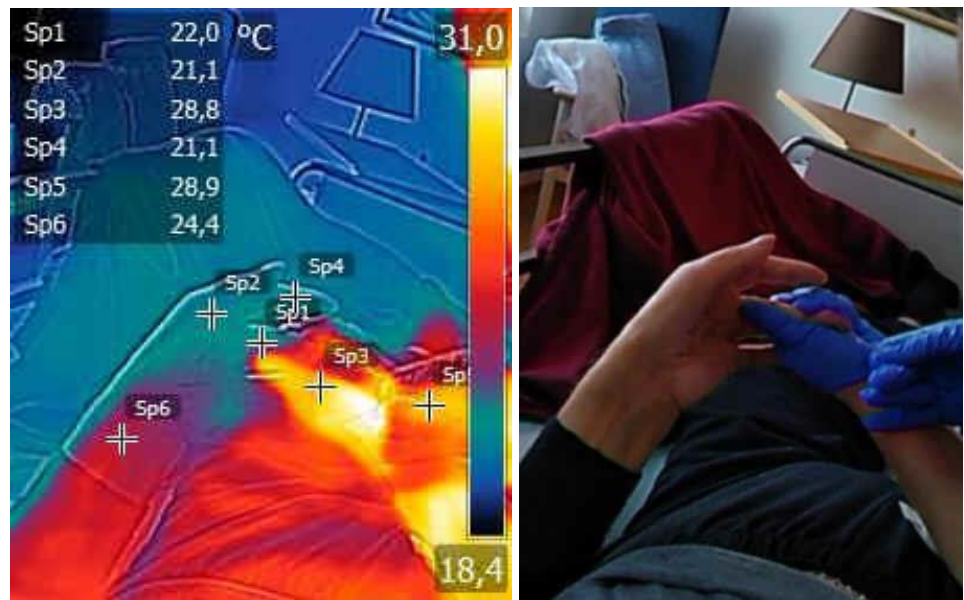

Figure 3. An example from blood sample extraction where the person of about 70 years of age has cold hands when compared with the hands of the sample taker (having blue gloves). The taking of blood sample often fails if having such cold hands and the hands need little rubbing or warming. Cold hands in normal room temperature suggests weak blood circulation in extremities seen often with older people.

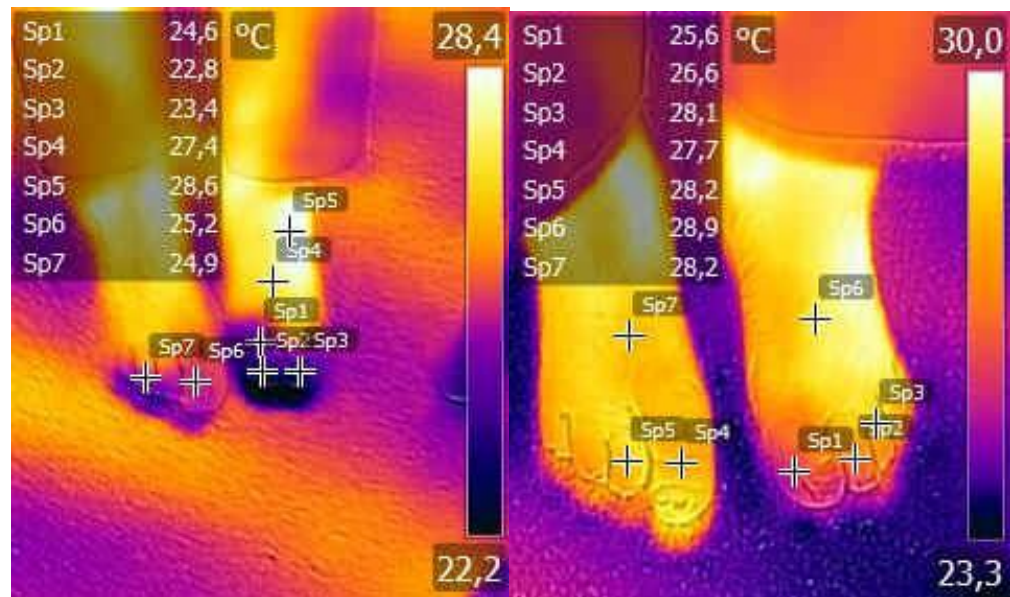

Figure 4. On the left side of the picture the blood circulation on toes has decreased due to walking without socks in the cold. On the right side of the picture socks have been used and the blood circulation has recovered. The person (aged 60 years) has signs of rheumatoid arthritis. Thermal camera can be used to demonstrate the decreased circulation in toes thus helping to explain the usefulness of the usage of socks. 


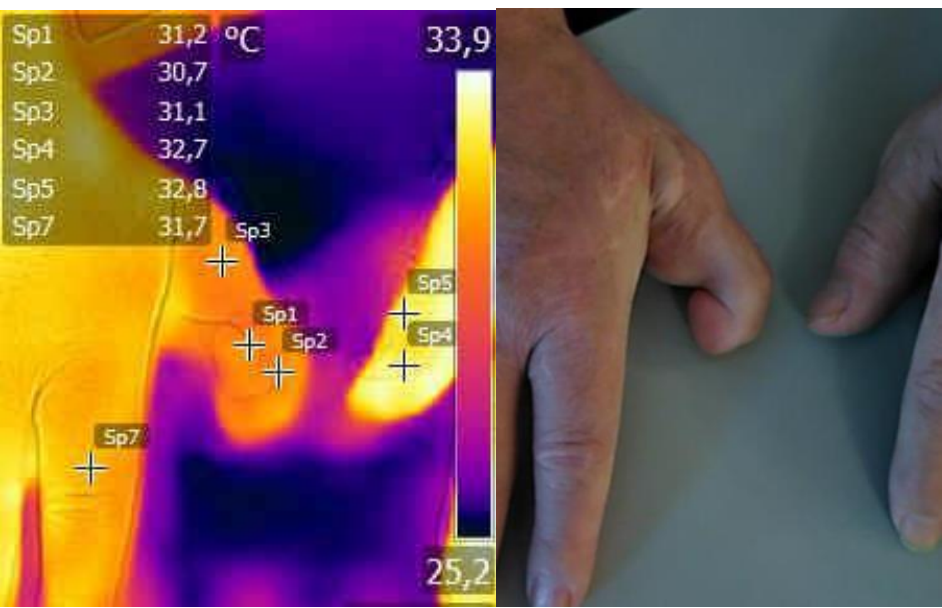

Figure 5. The person's thumb has rebuilt from a toe (Figure 4) and its blood circulation differs from the other thumb seen as deeper red shifting colour on the left side of the picture. On the right side picture a normal picture is taken simultaneously allowing the user to scrutinize both of the pictures.

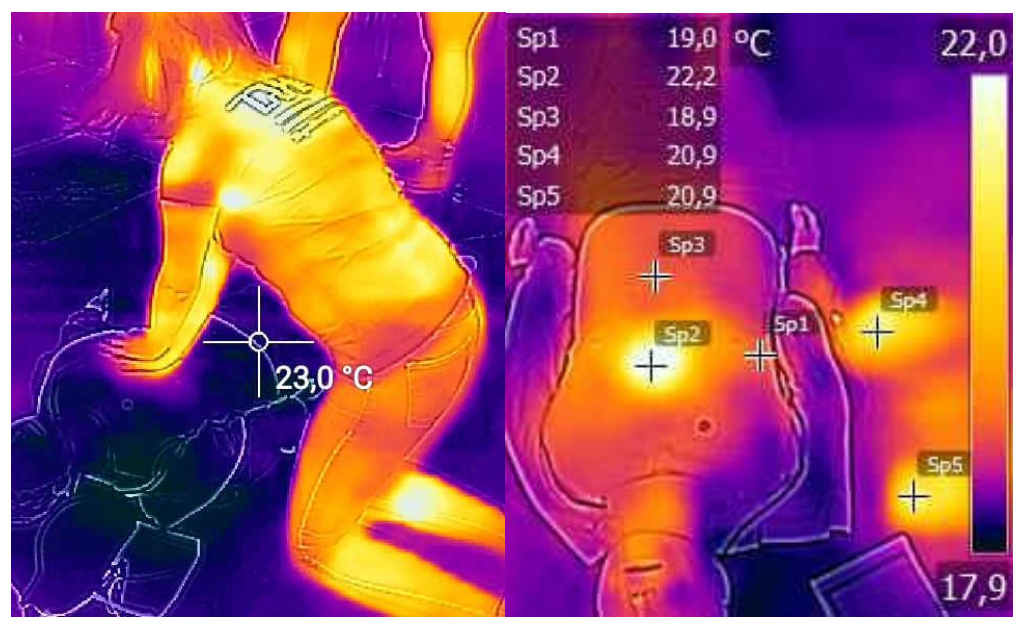

Figure 6. Recovery exercise with a doll. After several minutes, the warm traces from the palms and the kneecaps are seen in the resuscitation doll and in the ground. Correct position, and distance from the doll is easily seen from the warm traces. This example demonstrates the warm traces of a person which just left giving a hint of the time of departure.

\section{Discussion}

This is the pilot study utilizing by CAT S60 phone with inbuilt Flir Thermal camera to obtain patient information for diagnosis and treatment. One aim was to find out, if the sensitivity of the CAT S60 thermal camera would be well enough to help in nursing practices.
In nursing the thermal borders from limbs and from the whole body is usually obtained with thermometers needing contact with the skin. Thermal borders can be found also with a hand touching the skin. With a cellular phone having internal thermal camera thermal borders and the differences in limbs can be easily seen from a single picture. Bedsores due to long lasting immobiliza- 
tion of the patient could be possibly found with thermal camera. This is based on the work limitation in the muscles and respectively, a disturbance in the circulation of blood [2].

In figure 1 the whole area of a person is presented by the means of thermal imaging. This helps the physician to scrutinize larger skin areas to find temperature gradients [2]. Thermal imaging can be used to study skin or soft tissue diseases which are situated in the superficial skin layers [2]. In figure 2 some differences in heat dissipation were observed between right and left side of the back. Further studies may clarify if local vasculature, skin or muscle inflammations would be possible to find with a thermal camera; having decreased or increased heat traces. Deviated heat trace due to formerly surgical operation was seen in figure 5 . The amount of dissipated heat from limb (in this case the thumb) would possibly reveal the success of surgical operation or deviant vasculature in the rebuilt thumb. Thermal cam era would possibly help to evaluate the healing process after surgical operation; from the smaller part or from the whole body. The recovery of the blood circulation would be easily seen via heat dissipation making possible to compare limbs, toes or fingers. Also the progressive inflammation due to increased blood flow might be more visible observed via thermal pictures. In figure 3 the information of the temperature and thus the sufficient blood circulation in hands may help in saving capillaries and cuvettes thus reducing costs of the measurement when taking quick CRP. In figure 6 the warm trace (cooling down) of a human body is demonstrated. In recovery exercise the right pressing location on the sternum and the right distance from the resuscitation doll is easily located.

Different inflammations in the body and direct skin or inner structure lesions may produce heat differences; heat increase or decrease seen with thermal camera Especially in home care cases with a wound or some other skin lesion the usual procedure is that a practical nurse takes a picture and sends it to the nurse with comments about the patient. Then a nurse evaluates the picture and sends it to the geriatrician (specialized in elderly people) or to a physician. It would be an asset, if in the same picture, a combined $13 \mathrm{Mp}$ picture and a thermal camera picture is obtained including all thermal values. In the case of evolving inflammation, the progression of the state is easily seen in pictures. In home care, the cellular phones are used online to report to Hilkka application from home calls. RFID tags are used to control arrival and exit in home calls. Therefore, the usage of the cellular phones is familiar with the workers. The Flir Android application is very easy to use not needing any special knowledge. Due to the lack of skin injury thermal pictures, we need further studies to evaluate the usefulness of the CAT S60 phone to that purpose.

The property of the Flir software to show both normal and thermal image in the same picture one upon another allowing to separate pictures with a sweeping of a finger was extremely handy. As the CAT S60 with Flir software is very easy to use, it would be relatively easy to integrate the taking of thermal pictures into the daily routines in health care. Thermal imaging is suitable for mass screening revealing the state of the vasculature system non-invasively. It may suit expressing the state of the blockade or interference of the vasculature system seen as abnormal heat traces. The present preliminary study indicated that thermal imaging with CAT S60 might help in advancing nursing methods and offer some new usage targets in promoting the wellness of a patient.

\section{References}

[1] Deng F, Tang Q, Zheng Y, Zeng G, Zhong N. Infrared thermal imaging as a novel evaluation method for deep vein thrombosis in lower limbs. Med Phys. 2012 Dec;39(12):7224-7231.

https://doi.org/10.1118/1.4764485

[2] Cholewka A, Stanek A, Klimas A, Sieroń A, Drzazga Z. Thermal imaging application in chronic venous disease. Pilot study. J Therm Anal Calorim 2014;115(2):16091618. https://doi.org/10.1007/s10973-013-3356-0

[3] Mattsson N, Rönnemaa T, Juonala M, Viikari JS, Jokinen $E$, Hutri-Kähönen $N$, Kähönen $M$, Laitinen $T$, Raitakari OT. Arterial structure and function in young adults with the metabolic syndrome: The Cardiovascu- 
lar Risk in Young Finns Study. Eur Heart J. 2008 Mar;29(6):784-791.

https://doi.org/10.1093/eurheartj/ehm576

[4] García-Raso A, Llamas Sillero P. Elevated body fat is a risk factor for venous thromboembolism and thrombotic complications. Epidemiol Rep. 2014;2:3. https://doi.org/10.7243/2054-9911-2-3

[5] Jang MJ, Choi WI, Bang SM, Lee T, Kim YK, Ageno W, Oh D. Metabolic Syndrome Is Associated With Venous Thromboembolism in the Korean Population. Arterioscler Thromb Vasc Biol. 2009 Mar;29(3):311-315. https://doi.org/10.1161/ATVBAHA.109.184085

[6] Arora N, Martins D, Ruggerio D, Tousimis E, Swistel AJ, Osborne MP, Simmons RM. Effectiveness of a noninvasice digital infrared thermal imaging system in the detection of breast cancer. Am J Surg. 2008 Oct;196(4):523-526.

https://doi.org/10.1016/j.amjsurg.2008.06.015

[7] Lahiri BB, Bagavathiappan S, Jayakumar T, Philip J. Medical applications of infrared thermography: A review. Infrared Physics \& Technology 2012;55(4):221235. https://doi.org/10.1016/j.infrared.2012.03.007

[8] Kateb B, Yamamoto V, Yu C, Grundfest W, Gruen JP. Infrared thermal imaging: a review of the literature and case report. Neuroimage. 2009 Aug;47(Suppl 2):T154T162.

https://doi.org/10.1016/j.neuroimage.2009.03.043

[9] Tan JH, Ng EYK, Rajendra Acharya U, Chee C. Infrared thermography on ocular surface temperature: $A$ review. Infrared Physics \& Technology 2009;52(4):97108. https://doi.org/10.1016/j.infrared.2009.05.002

[10] Sand O, Sjaastad OV, Haug E, Bjalie JG. 'Ihminen. Fysiologia ja anatomia'. Sanoma Pro Oy; 2011. pp. 444449.

[11] Hess KL, Wilson TE, Sauder CL, Gao Z, Ray CA, Monahan KD. Aging affects the cardiovascular responses to cold stress in humans. J Appl Physiol (1985). 2009 Oct;107(4):1076-1082.

[12] Koljonen V, Väänänen U, Isoherranen K. Mikä on lääkärin rooli haavanhoidossa. Suomen Lääkärilehti 8/2017 VSK 72, 484-485.

[13] Laukkanen T, Khan H, Zaccardi F, Laukkanen JA. Association between sauna bathing and fatal cardiovascular and all-cause mortality events. JAMA Intern Med. 2015 Apr;175(4):542-548. https://doi.org/10.1001/jamainternmed.2014.8187 